\title{
Iron availability and outcomes in critical illness
}

\section{To the Editor:}

We read with interest the recent paper from Tacke and colleagues (1), in which they report the measurement of multiple serum iron parameters in a cohort of patients admitted to the intensive care unit (ICU). Their principal finding was the positive correlation of transferrin saturation on admission with short- and long-term mortality. The authors suggest that elevated serum iron availability may worsen prognosis in critical illness, possibly through the production of reactive oxygen species and/or the increased supply of iron to invading microorganisms, and they call for prospective trials of iron chelators in critical illness and sepsis.

We would like to make two brief comments on this work. The first relates to the utility of transferrin saturation as an index of iron availability in patients with critical illness. As acknowledged by the authors, serum transferrin typically falls substantially in septic patients, reflecting its nature as a negative acute phase protein. The assumption that calculated transferrin saturation remains proportional to tissue iron availability in the context of low transferrin and hypoferremia may not be correct. Interestingly, a previous ICU study using an index of iron availability (red cell hypochromasia) based on tissue utilisation rather than serum iron parameters reported that iron deficiency, rather than increased iron availability, was associated with adverse outcomes (2).

Our second point relates to the use of iron chelators in patients with sepsis and/or critical illness. Although this strategy may theoretically impede microbial 
growth, the notion of a direct link between iron availability and infection in critical illness has been challenged by recent studies. For example, the use of repeated doses of intravenous iron in ICU trauma patients was not associated with an increase in the rate or severity of infection, compared with placebo (3). Moreover, any potential benefits of iron chelation would need to be weighed against the potential for increases in pulmonary vascular resistance and right ventricular afterload (4). Several observations suggest that this effect would be a significant concern. First, the iron chelator desferrioxamine is known to induce modest pulmonary vasoconstriction in healthy volunteers, possibly by activating the hypoxia-inducible factor transcriptional pathway (5). Second, in patients with chronic pulmonary hypertension at high altitude, depletion of iron stores was observed to increase systolic pulmonary artery pressure (6). Third, in otherwise healthy adults with iron deficiency, the pulmonary hypertensive response to acute hypoxia is markedly enhanced, and can be greatly attenuated by intravenous iron supplementation (7).

In combination with the recent findings of Tacke et al, these observations suggest that the relationship between iron availability and outcome in ICU is likely to be complex, and bring into focus the difficulty of assessing iron status in patients with critical illness. Ongoing clinical trials of intravenous iron in ICU, such as the IRONMAN trial, may shed more light on this important issue.

\author{
Nick P Talbot, ${ }^{1,2}$ Matthew C Frise, ${ }^{2}$ Thomas G Smith ${ }^{2,3}$ \\ ${ }^{1}$ Nuffield Department of Medicine, University of Oxford, United Kingdom \\ ${ }^{2}$ Department of Physiology, Anatomy \& Genetics, University of Oxford, United Kingdom \\ ${ }^{3}$ Nuffield Division of Anaesthetics, University of Oxford, United Kingdom
}




\section{References}

1. Tacke F, Nuraldeen R, Koch A, et al. Iron parameters determine the prognosis of critically ill patients. Crit Care Med 2016 doi: 10.1097/CCM.0000000000001607.

2. Bellamy MC \& Gedney JA. Unrecognised iron deficiency in critical illness. Lancet 1998; 352(9144):1903

3. Pieracci FM, Stovall RT, Jaouen B, et al. A multicentre, randomized clinical trial of IV iron supplementation for anemia of traumatic critical illness. Crit Care Med 2015; 42(9):2048-2057

4. Price LC, Wort SJ, Finney SJ, et al. Pulmonary vascular and right ventricular dysfunction in adult critical care: current and emerging options for management: a systematic literature review. Crit Care 2010; 14(5):R169

5. Balanos GM, Dorrington KL, Robbins PA. Desferrioxamine elevates pulmonary vascular resistance in humans: potential for involvement of HIF-1. J Appl Physiol 2002; 92(6):2501-2507

6. Smith TG, Talbot NP, Privat C, et al. Effects of iron supplementation and depletion on hypoxic pulmonary hypertension: two randomized controlled trials. JAMA 2009; 302(13):1444-1450

7. Frise MC, Cheng HY, Nickol AH, et al. Clinical iron deficiency disturbs normal human responses to hypoxia. J Clin Invest 2016. In press. 